\title{
SOME PROPERTIES OF MAXIMAL OPEN SETS
}

\author{
FUMIE NAKAOKA and NOBUYUKI ODA
}

\author{
Received 11 July 2002
}

\begin{abstract}
Some fundamental properties of maximal open sets are obtained, such as decomposition theorem for a maximal open set. Basic properties of intersections of maximal open sets are established, such as the law of radical closure.
\end{abstract}

2000 Mathematics Subject Classification: 54A05, 54D99.

1. Introduction. A proper nonempty open subset $U$ of a topological space $X$ is said to be a maximal open set if any open set which contains $U$ is $X$ or $U$. In [2], we study minimal open sets. Although the definition of the maximal open set is obtained by "dualizing" the definition of the minimal open set, the properties of them are quite different, as we see in this paper, especially the results in the last two sections. The purpose of this paper is to prove some fundamental properties of maximal open sets and establish a part of the foundation of the theory of maximal open sets in topological spaces.

In Section 2, we prove some basic results which are necessary for the subsequent arguments. We obtain a relation among maximal open sets in Theorem 2.5. At the end of this section, we show that for any proper nonempty cofinite open subset $V$, there exists, at least, one maximal open set $U$ which contains $V$ (Theorem 2.7).

In Section 3, we study some relations among closure, interior, and maximal open sets. As an application, we prove a result about a preopen set (Theorem 3.11).

Let $U=\left\{U_{\lambda} \mid \lambda \in \Lambda\right\}$ be a set of some maximal open sets $U_{\lambda}$. Then, we refer to the intersection $\cap \mathcal{U}=\cap_{\lambda \in \Lambda} U_{\lambda}$ as the radical of $\mathcal{U}$. In the last two sections, we study various properties of radicals.

In Section 4, we prove fundamental properties of radicals of maximal open sets. We establish a very useful decomposition theorem for a maximal open set in Theorem 4.7. Theorem 4.7 will be applied to prove Theorem 4.8. Theorem 4.9 gives a sufficient condition for the set of all maximal open sets. In the rest of this section, we study the case when radicals are closed sets.

In Section 5, we consider the closure of the radicals of maximal open sets. We establish "The law of radical closure" in Theorem 5.4.

2. Maximal open sets. Let $(X, \tau)$ be a topological space. 
DEFINITION 2.1. A proper nonempty open subset $U$ of $X$ is said to be a maximal open set if any open set which contains $U$ is $X$ or $U$.

LEMMA 2.2. (1) Let $U$ be a maximal open set and $W$ an open set. Then, $U \cup$ $W=X$ or $W \subset U$.

(2) Let $U$ and $V$ be maximal open sets. Then, $U \cup V=X$ or $U=V$.

Proof. (1) Let $W$ be an open set such that $U \cup W \neq X$. Since $U$ is a maximal open set and $U \subset U \cup W$, we have $U \cup W=U$. Therefore, $W \subset U$.

(2) If $U \cup V \neq X$, then $U \subset V$ and $V \subset U$ by (1). Therefore $U=V$.

Proposition 2.3. Let $U$ be a maximal open set. If $x$ is an element of $U$, then for any open neighborhood $W$ of $x, W \cup U=X$ or $W \subset U$.

Proof. By Lemma 2.2(1), we have the result.

THEOREM 2.4. Let $U_{\alpha}, U_{\beta}$, and $U_{\gamma}$ be maximal open sets such that $U_{\alpha} \neq U_{\beta}$. If $U_{\alpha} \cap U_{\beta} \subset U_{\gamma}$, then $U_{\alpha}=U_{\gamma}$ or $U_{\beta}=U_{\gamma}$.

Proof. We see that

$$
\begin{aligned}
U_{\alpha} \cap U_{\gamma} & =U_{\alpha} \cap\left(U_{\gamma} \cap X\right) \\
& =U_{\alpha} \cap\left(U_{\gamma} \cap\left(U_{\alpha} \cup U_{\beta}\right)\right) \quad(\text { by Lemma 2.2(2)) } \\
& =U_{\alpha} \cap\left(\left(U_{\gamma} \cap U_{\alpha}\right) \cup\left(U_{\gamma} \cap U_{\beta}\right)\right) \\
& =\left(U_{\alpha} \cap U_{\gamma}\right) \cup\left(U_{\gamma} \cap U_{\alpha} \cap U_{\beta}\right) \\
& =\left(U_{\alpha} \cap U_{\gamma}\right) \cup\left(U_{\alpha} \cap U_{\beta}\right) \quad\left(\text { by } U_{\alpha} \cap U_{\beta} \subset U_{\gamma}\right) \\
& =U_{\alpha} \cap\left(U_{\gamma} \cup U_{\beta}\right) .
\end{aligned}
$$

Hence we have $U_{\alpha} \cap U_{\gamma}=U_{\alpha} \cap\left(U_{\gamma} \cup U_{\beta}\right)$. If $U_{\gamma} \neq U_{\beta}$, then $U_{\gamma} \cup U_{\beta}=X$, and hence $U_{\alpha} \cap U_{\gamma}=U_{\alpha}$; namely, $U_{\alpha} \subset U_{\gamma}$. Since $U_{\alpha}$ and $U_{\gamma}$ are maximal open sets, we have $U_{\alpha}=U_{\gamma}$.

THEOREM 2.5. Let $U_{\alpha}, U_{\beta}$, and $U_{\gamma}$ be maximal open sets, which are different from each other. Then,

$$
U_{\alpha} \cap U_{\beta} \not \subset U_{\alpha} \cap U_{\gamma}
$$

ProoF. If $U_{\alpha} \cap U_{\beta} \subset U_{\alpha} \cap U_{\gamma}$, then we see that

$$
\left(U_{\alpha} \cap U_{\beta}\right) \cup\left(U_{\beta} \cap U_{\gamma}\right) \subset\left(U_{\alpha} \cap U_{\gamma}\right) \cup\left(U_{\beta} \cap U_{\gamma}\right)
$$

hence,

$$
U_{\beta} \cap\left(U_{\alpha} \cup U_{\gamma}\right) \subset\left(U_{\alpha} \cup U_{\beta}\right) \cap U_{\gamma} .
$$

Since $U_{\alpha} \cup U_{\gamma}=X=U_{\alpha} \cup U_{\beta}$, we have $U_{\beta} \subset U_{\gamma}$. It follows that $U_{\beta}=U_{\gamma}$, which contradicts our assumption. 
Proposition 2.6. Let $U$ be a maximal open set and $x$ an element of $U$. Then,

$$
U=\cup\{W \mid W \text { is an open neighborhood of } x \text { such that } W \cup U \neq X\} \text {. }
$$

Proof. By Proposition 2.3 and the fact that $U$ is an open neighborhood of $x$, we have

$U \subset \cup\{W \mid W$ is an open neighborhood of $x$ such that $W \cup U \neq X\} \subset U$. (2.6)

Therefore, we have the result.

Finally, we prove an existence theorem of maximal open sets for special cases. We refer to the complement of any finite subset as a cofinite subset.

THEOREM 2.7. Let $V$ be a proper nonempty cofinite open subset. Then, there exists, at least, one (cofinite) maximal open set $U$ such that $V \subset U$.

Proof. If $V$ is a maximal open set, we may set $U=V$. If $V$ is not a maximal open set, then there exists an (cofinite) open set $V_{1}$ such that $V \subsetneq V_{1} \neq X$. If $V_{1}$ is a maximal open set, we may set $U=V_{1}$. If $V_{1}$ is not a maximal open set, then there exists an (cofinite) open set $V_{2}$ such that $V \subsetneq V_{1} \subsetneq V_{2} \neq X$. Continuing this process, we have a sequence of open sets

$$
V \subsetneq V_{1} \subsetneq V_{2} \cdots \subsetneq V_{k} \subsetneq \cdots
$$

Since $V$ is a cofinite set, this process repeats only finitely. Then, finally, we get a maximal open set $U=V_{n}$ for some positive integer $n$.

3. Closure, interior, and maximal open sets. We begin with the following theorem.

THEOREM 3.1. Let $U$ be a maximal open set and $x$ an element of $X-U$. Then, $X-U \subset W$ for any open neighborhood $W$ of $x$.

Proof. Since $x \in X-U$, we have $W \not \subset U$ for any open neighborhood $W$ of $x$. Then, $W \cup U=X$ by Lemma 2.2(1). Therefore, $X-U \subset W$.

COROLlaRY 3.2. Let $U$ be a maximal open set. Then, either of the following (1) and (2) holds:

(1) for each $x \in X-U$ and each open neighborhood $W$ of $x, W=X$;

(2) there exists an open set $W$ such that $X-U \subset W$ and $W \subsetneq X$.

Proof. If (1) does not hold, then there exists an element $x$ of $X-U$ and an open neighborhood $W$ of $x$ such that $W \subsetneq X$. By Theorem 3.1, we have $X-U \subset W$.

COROLLARY 3.3. Let $U$ be a maximal open set. Then, either of the following (1) and (2) holds: 
(1) for each $x \in X-U$ and each open neighborhood $W$ of $x$, we have $X-U \subsetneq$ $W$;

(2) there exists an open set $W$ such that $X-U=W \neq X$.

Proof. Assume that (2) does not hold. Then, by Theorem 3.1, we have $X-$ $U \subset W$ for each $x \in X-U$ and each open neighborhood $W$ of $x$. Hence, we have $X-U \subsetneq W$.

THeOREM 3.4. Let $U$ be a maximal open set. Then, $\mathrm{Cl}(U)=X$ or $\mathrm{Cl}(U)=U$.

Proof. Since $U$ is a maximal open set, only the following cases (1) and (2) occur by Corollary 3.3:

(1) for each $x \in X-U$ and each open neighborhood $W$ of $x$, we have $X-U \subsetneq$ $W$ : let $x$ be any element of $X-U$ and $W$ any open neighborhood of $x$. Since $X-U \neq W$, we have $W \cap U \neq \varnothing$ for any open neighborhood $W$ of $x$. Hence, $X-U \subset \mathrm{Cl}(U)$. Since $X=U \cup(X-U) \subset U \cup \mathrm{Cl}(U)=\mathrm{Cl}(U) \subset X$, we have $\mathrm{Cl}(U)=X$;

(2) there exists an open set $W$ such that $X-U=W \neq X$ : since $X-U=W$ is an open set, $U$ is a closed set. Therefore, $U=\mathrm{Cl}(U)$.

THeOREM 3.5. Let $U$ be a maximal open set. Then, $\operatorname{Int}(X-U)=X-U$ or $\operatorname{Int}(X-U)=\varnothing$.

Proof. By Corollary 3.3, we have either (1) $\operatorname{Int}(X-U)=\varnothing$ or (2) $\operatorname{Int}(X-$ $U)=X-U$.

THEOREM 3.6. Let $U$ be a maximal open set and $S$ a nonempty subset of $X-U$. Then, $\operatorname{Cl}(S)=X-U$.

Proof. Since $\varnothing \neq S \subset X-U$, we have $W \cap S \neq \varnothing$ for any element $x$ of $X-U$ and any open neighborhood $W$ of $x$ by Theorem 3.1. Then, $X-U \subset \mathrm{Cl}(S)$. Since $X-U$ is a closed set and $S \subset X-U$, we see that $\mathrm{Cl}(S) \subset \mathrm{Cl}(X-U)=X-U$. Therefore, $X-U=\mathrm{Cl}(S)$.

COROLlary 3.7. Let $U$ be a maximal open set and $M$ a subset of $X$ with $U \subsetneq M$. Then, $\operatorname{Cl}(M)=X$.

Proof. Since $U \subsetneq M \subset X$, there exists a nonempty subset $S$ of $X-U$ such that $M=U \cup S$. Hence, we have $\mathrm{Cl}(M)=\mathrm{Cl}(S \cup U)=\mathrm{Cl}(S) \cup \mathrm{Cl}(U) \supset(X-U) \cup$ $U=X$ by Theorem 3.6. Therefore, $\mathrm{Cl}(M)=X$.

THEOREM 3.8. Let $U$ be a maximal open set and assume that the subset $X-U$ has two elements at least. Then, $\mathrm{Cl}(X-\{a\})=X$ for any element $a$ of $X-U$.

Proof. Since $U \subsetneq X-\{a\}$ by our assumption, we have the result by Corollary 3.7.

THEOREM 3.9. Let $U$ be a maximal open set and $N$ a proper subset of $X$ with $U \subset N$. Then, $\operatorname{Int}(N)=U$. 
Proof. If $N=U$, then $\operatorname{Int}(N)=\operatorname{Int}(U)=U$. Otherwise $N \neq U$, and hence $U \subsetneq N$. It follows that $U \subset \operatorname{Int}(N)$. Since $U$ is a maximal open set, we have also $\operatorname{Int}(N) \subset U$. Therefore, $\operatorname{Int}(N)=U$.

THEOREM 3.10. Let $U$ be a maximal open set and $S$ a nonempty subset of $X-U$. Then,

$$
X-\mathrm{Cl}(S)=\operatorname{Int}(X-S)=U
$$

Proof. Since $U \subset X-S \subsetneq X$ by our assumption, we have the result by Theorems 3.6 and 3.9 .

A subset $M$ of a space $(X, \tau)$ is called a preopen set if $M \subset \operatorname{IntCl}(M)$. Then, Corollary 3.7 implies the following result.

THEOREM 3.11. Let $U$ be a maximal open set and $M$ any subset of $X$ with $U \subset M$. Then, $M$ is a preopen set.

Proof. If $M=U$, then $M$ is an open set. Therefore, $M$ is a preopen set. Otherwise, $U \subsetneq M$, then $\operatorname{Int} \mathrm{Cl}(M)=\operatorname{Int} X=X \supset M$ by Corollary 3.7. Therefore, $M$ is a preopen set.

COROLlary 3.12. Let $U$ be a maximal open set. Then, $X-\{a\}$ is a preopen set for any element $a$ of $X-U$.

Proof. Since $U \subset X-\{a\}$ by our assumption, we have the result by Theorem 3.11.

\section{Fundamental properties of radicals}

DEFINITION 4.1. Let $U_{\lambda}$ be a maximal open set for any element $\lambda$ of $\Lambda$. Let $u=\left\{U_{\lambda} \mid \lambda \in \Lambda\right\} ; \cap U=\cap_{\lambda \in \Lambda} U_{\lambda}$ is called the radical of $u$.

The intersection of all maximal ideals of a ring $\mathscr{R}$ is called the (Jacobson) radical of $\mathscr{R}[1,3]$. Following this terminology in the theory of rings, we use the terminology "radical" for the intersection of maximal open sets.

The symbol $\Lambda \backslash \Gamma$ means difference of index sets; namely, $\Lambda \backslash \Gamma=\Lambda-\Gamma$, and the cardinality of a set $\Lambda$ is denoted by $|\Lambda|$ in the following arguments.

THEOREM 4.2. Assume that $|\Lambda| \geq 2$. Let $U_{\lambda}$ be a maximal open set for any element $\lambda$ of $\Lambda$ and $U_{\lambda} \neq U_{\mu}$ for any elements $\lambda$ and $\mu$ of $\Lambda$ with $\lambda \neq \mu$.

(1) Let $\mu$ be any element of $\Lambda$. Then, $X-\cap_{\lambda \in \Lambda \backslash\{\mu\}} U_{\lambda} \subset U_{\mu}$.

(2) Let $\mu$ be any element of $\Lambda$. Then, $\cap_{\lambda \in \Lambda \backslash\{\mu\}} U_{\lambda} \neq \varnothing$.

Proof. Let $\mu$ be any element of $\Lambda$. (1) By Lemma 2.2(2), we have $X-U_{\mu} \subset U_{\lambda}$ for any element $\lambda$ of $\Lambda$ with $\lambda \neq \mu$. Then, $X-U_{\mu} \subset \cap_{\lambda \in \Lambda \backslash\{\mu\}} U_{\lambda}$. Therefore, we have $X-\cap_{\lambda \in \Lambda \backslash\{\mu\}} U_{\lambda} \subset U_{\mu}$.

(2) If $\cap_{\lambda \in \Lambda \backslash\{\mu\}} U_{\lambda}=\varnothing$, we have $X=U_{\mu}$ by (1). This contradicts our assumption that $U_{\mu}$ is a maximal open set. Therefore, we have $\cap_{\lambda \in \Lambda \backslash\{\mu\}} U_{\lambda} \neq \varnothing$. 
COROLLARY 4.3. Let $U_{\lambda}$ be a maximal open set for any element $\lambda$ of $\Lambda$ and $U_{\lambda} \neq U_{\mu}$ for any elements $\lambda$ and $\mu$ of $\Lambda$ with $\lambda \neq \mu$. If $|\Lambda| \geq 3$, then $U_{\lambda} \cap U_{\mu} \neq \varnothing$ for any elements $\lambda$ and $\mu$ of $\Lambda$ with $\lambda \neq \mu$.

Proof. By Theorem 4.2(2), we have the result.

THEOREM 4.4. Let $U_{\lambda}$ be a maximal open set for any element $\lambda$ of $\Lambda$ and $U_{\lambda} \neq U_{\mu}$ for any elements $\lambda$ and $\mu$ of $\Lambda$ with $\lambda \neq \mu$. Assume that $|\Lambda| \geq 2$. Let $\mu$ be any element of $\Lambda$. Then, $\cap_{\lambda \in \Lambda \backslash\{\mu\}} U_{\lambda} \not \subset U_{\mu} \not \subset \cap_{\lambda \in \Lambda \backslash\{\mu\}} U_{\lambda}$.

Proof. Let $\mu$ be any element of $\Lambda$. If $\cap_{\lambda \in \Lambda \backslash\{\mu\}} U_{\lambda} \subset U_{\mu}$, then we see that $X=\left(X-\cap_{\lambda \in \Lambda \backslash\{\mu\}} U_{\lambda}\right) \cup \cap_{\lambda \in \Lambda \backslash\{\mu\}} U_{\lambda} \subset U_{\mu}$ by Theorem 4.2(1). This contradicts our assumption. If $U_{\mu} \subset \cap_{\lambda \in \Lambda \backslash\{\mu\}} U_{\lambda}$, then we have $U_{\mu} \subset U_{\lambda}$, and hence $U_{\mu}=U_{\lambda}$ for any element $\lambda$ of $\Lambda \backslash\{\mu\}$. This contradicts our assumption that $U_{\mu} \neq U_{\lambda}$ when $\lambda \neq \mu$.

COROLLARY 4.5. Let $U_{\lambda}$ be a maximal open set for any element $\lambda$ of $\Lambda$ and $U_{\lambda} \neq U_{\mu}$ for any elements $\lambda$ and $\mu$ of $\Lambda$ with $\lambda \neq \mu$. If $\Gamma$ is a proper nonempty subset of $\Lambda$, then $\cap_{\lambda \in \Lambda \backslash \Gamma} U_{\lambda} \not \subset \cap_{\gamma \in \Gamma} U_{\gamma} \not \subset \cap_{\lambda \in \Lambda \backslash \Gamma} U_{\lambda}$.

Proof. Let $\gamma$ be any element of $\Gamma$. We see $\cap_{\lambda \in \Lambda \mid \Gamma} U_{\lambda}=\cap_{\lambda \in((\Lambda \mid \Gamma) \cup\{\gamma\}) \backslash\{\gamma\}} U_{\lambda} \not \subset$ $U_{\gamma}$ by Theorem 4.4. Therefore we see $\cap_{\lambda \in \Lambda \mid \Gamma} U_{\lambda} \not \subset \cap_{\gamma \in \Gamma} U_{\gamma}$. On the other hand, since $\cap_{\gamma \in \Gamma} U_{\gamma}=\cap_{\gamma \in \Lambda \backslash(\Lambda \backslash \Gamma)} U_{\gamma} \not \subset \cap_{\lambda \in \Lambda \backslash \Gamma} U_{\lambda}$, we have $\cap_{\gamma \in \Gamma} U_{\gamma} \not \subset \cap_{\lambda \in \Lambda \backslash \Gamma} U_{\lambda}$.

THEOREM 4.6. Let $U_{\lambda}$ be a maximal open set for any element $\lambda$ of $\Lambda$ and $U_{\lambda} \neq U_{\mu}$ for any elements $\lambda$ and $\mu$ of $\Lambda$ with $\lambda \neq \mu$. If $\Gamma$ is a proper nonempty subset of $\Lambda$, then $\cap_{\lambda \in \Lambda} U_{\lambda} \subsetneq \cap_{\gamma \in \Gamma} U_{\gamma}$.

Proof. By Corollary 4.5 , we have $\cap_{\lambda \in \Lambda} U_{\lambda}=\left(\cap_{\lambda \in \Lambda \mid \Gamma} U_{\lambda}\right) \cap\left(\cap_{\gamma \in \Gamma} U_{\gamma}\right) \subsetneq$ $\cap_{\gamma \in \Gamma} U_{\gamma}$.

THEOREM 4.7 (a decomposition theorem for maximal open set). Assume that $|\Lambda| \geq 2$. Let $U_{\lambda}$ be a maximal open set for any element $\lambda$ of $\Lambda$ and $U_{\lambda} \neq U_{\mu}$ for any elements $\lambda$ and $\mu$ of $\Lambda$ with $\lambda \neq \mu$. Then, for any element $\mu$ of $\Lambda$,

$$
U_{\mu}=\left(\cap_{\lambda \in \Lambda} U_{\lambda}\right) \cup\left(X-\cap_{\lambda \in \Lambda \backslash\{\mu\}} U_{\lambda}\right) .
$$

Proof. Let $\mu$ be an element of $\Lambda$. By Theorem 4.2(1), we have

$$
\begin{aligned}
\left(\cap_{\lambda \in \Lambda} U_{\lambda}\right) \cup\left(X-\cap_{\lambda \in \Lambda \backslash\{\mu\}} U_{\lambda}\right)= & \left(\left(\cap_{\lambda \in \Lambda \backslash\{\mu\}} U_{\lambda}\right) \cap U_{\mu}\right) \cup\left(X-\cap_{\lambda \in \Lambda \backslash\{\mu\}} U_{\lambda}\right) \\
= & \left(\left(\cap_{\lambda \in \Lambda \backslash\{\mu\}} U_{\lambda}\right) \cup\left(X-\cap_{\lambda \in \Lambda \backslash\{\mu\}} U_{\lambda}\right)\right) \\
& \cap\left(U_{\mu} \cup\left(X-\cap_{\lambda \in \Lambda \backslash\{\mu\}} U_{\lambda}\right)\right) \\
= & U_{\mu} \cup\left(X-\cap_{\lambda \in \Lambda \backslash\{\mu\}} U_{\lambda}\right)=U_{\mu} .
\end{aligned}
$$

Therefore, we have $U_{\mu}=\left(\cap_{\lambda \in \Lambda} U_{\lambda}\right) \cup\left(X-\cap_{\lambda \in \Lambda \backslash\{\mu\}} U_{\lambda}\right)$. 
THEOREM 4.8. Let $U_{\lambda}$ be a maximal open set for any element $\lambda$ of a finite set $\Lambda$ and $U_{\lambda} \neq U_{\mu}$ for any elements $\lambda$ and $\mu$ of $\Lambda$ with $\lambda \neq \mu$. If $\cap_{\lambda \in \Lambda} U_{\lambda}$ is a closed set, then $U_{\lambda}$ is a closed set for any element $\lambda$ of $\Lambda$.

Proof. By Theorem 4.7, we have $U_{\mu}=\left(\cap_{\lambda \in \Lambda} U_{\lambda}\right) \cup\left(X-\cap_{\lambda \in \Lambda \backslash\{\mu\}} U_{\lambda}\right)=$ $\left(\cap_{\lambda \in \Lambda} U_{\lambda}\right) \cup\left(\cup_{\lambda \in \Lambda \backslash\{\mu\}}\left(X-U_{\lambda}\right)\right)$. Since $\Lambda$ is a finite set, we see that $\cup_{\lambda \in \Lambda \backslash\{\mu\}}(X-$ $\left.U_{\lambda}\right)$ is a closed set. Hence, $U_{\mu}$ is a closed set by our assumption.

As an application of Theorem 4.7, we give another proof of Theorem 4.6.

ANOTHER PROOF OF THEOREM 4.6. Since $\Lambda \supsetneq \Gamma \neq \varnothing$, there exists an element $v$ of $\Lambda$ such that $v \notin \Gamma$ and an element $\mu$ of $\Gamma$. If $|\Gamma|=1$, then we have $\cap_{\lambda \in \Lambda} U_{\lambda} \subset U_{\mu}$. If $\cap_{\lambda \in \Lambda} U_{\lambda}=U_{\mu}$, then we have $U_{\mu} \subset U_{\lambda}$ for any element $\lambda$ of $\Lambda$. Since $U_{\lambda}$ is a maximal open set for any element $\lambda$ of $\Lambda$, we have $U_{\mu}=U_{\lambda}$, which contradicts our assumption. Hence, we have $\cap_{\lambda \in \Lambda} U_{\lambda} \subsetneq U_{\mu}$. If $|\Gamma| \geq 2$, then by Theorem 4.7, we have

$$
\begin{aligned}
& U_{v}=\left(\cap_{\lambda \in \Lambda} U_{\lambda}\right) \cup\left(X-\cap_{\lambda \in \Lambda \backslash\{v\}} U_{\lambda}\right), \\
& U_{\mu}=\left(\cap_{\gamma \in \Gamma} U_{\gamma}\right) \cup\left(X-\cap_{\gamma \in \Gamma \backslash\{\mu\}} U_{\gamma}\right) .
\end{aligned}
$$

If $\cap_{\lambda \in \Lambda} U_{\lambda}=\cap_{\gamma \in \Gamma} U_{\gamma}$, then $\cap_{\gamma \in \Gamma} U_{\gamma}=\cap_{\lambda \in \Lambda} U_{\lambda} \subset \cap_{\lambda \in \Lambda \backslash\{v\}} U_{\lambda} \subset \cap_{\gamma \in \Gamma} U_{\gamma}$. Hence, we have $\cap_{\lambda \in \Lambda \backslash\{v\}} U_{\lambda}=\cap_{\gamma \in \Gamma} U_{\gamma}$. Therefore, $\cap_{\lambda \in \Lambda \backslash\{v\}} U_{\lambda}=\cap_{\gamma \in \Gamma} U_{\gamma} \subset \cap_{\gamma \in \Gamma \backslash\{\mu\}} U_{\gamma}$. Hence, we see that $U_{v} \supset U_{\mu}$. It follows that $U_{v}=U_{\mu}$ with $\nu \neq \mu$. This contradicts our assumption.

THEOREM 4.9. Assume that $|\Lambda| \geq 2$. Let $U_{\lambda}$ be a maximal open set for any element $\lambda$ of $\Lambda$ and $U_{\lambda} \neq U_{\mu}$ for any elements $\lambda$ and $\mu$ of $\Lambda$ with $\lambda \neq \mu$. If $\cap_{\lambda \in \Lambda} U_{\lambda}=\varnothing$, then $\left\{U_{\lambda} \mid \lambda \in \Lambda\right\}$ is the set of all maximal open sets of $X$.

Proof. If there exists another maximal open set $U_{v}$ of $X$, which is not equal to $U_{\lambda}$ for any element $\lambda$ of $\Lambda$, then $\varnothing=\cap_{\lambda \in \Lambda} U_{\lambda}=\cap_{\lambda \in(\Lambda \cup\{v\}) \backslash\{v\}} U_{\lambda}$. By Theorem 4.2(2), we see that $\cap_{\lambda \in(\Lambda \cup\{v\}) \backslash\{v\}} U_{\lambda} \neq \varnothing$. This contradicts our assumption.

EXAMPLE 4.10. If each point $\{x\}$ is closed (e.g., $X$ is a Hausdorff space or a cofinite space or a cocountable space), then $X-\{a\}$ is a maximal open set for any element $a$ of $X$. Moreover, we see that $\{X-\{a\} \mid a \in X\}$ is the set of all maximal open sets of $X$ by Theorem 4.9, since $\cap_{a \in X}(X-\{a\})=\varnothing$.

Proposition 4.11. Let $A$ and $B$ be subsets of $X$. If $A \cup B=X, A \cap B$ is a closed set, and $A$ is an open set, then $B$ is a closed set.

Proof. Since $X-A \subset B$, then we see that

$$
(A \cap B) \cup(X-A)=(A \cup(X-A)) \cap(B \cup(X-A))=B \cup(X-A)=B .
$$

Since $A \cap B$ and $X-A$ are closed sets, we see that $B$ is a closed set. 
Proposition 4.12. Let $U_{\lambda}$ be an open set for any element $\lambda$ of $\Lambda$ and $U_{\lambda} \cup$ $U_{\mu}=X$ for any elements $\lambda$ and $\mu$ of $\Lambda$ with $\lambda \neq \mu$. If $\cap_{\lambda \in \Lambda} U_{\lambda}$ is a closed set, then $\cap_{\lambda \in \Lambda \backslash\{\mu\}} U_{\lambda}$ is a closed set for any element $\mu$ of $\Lambda$.

Proof. Let $\mu$ be any element of $\Lambda$. Since $U_{\lambda} \cup U_{\mu}=X$ for any element $\lambda$ of $\Lambda$ with $\lambda \neq \mu$, we have

$$
U_{\mu} \cup\left(\cap_{\lambda \in \Lambda \backslash\{\mu\}} U_{\lambda}\right)=\cap_{\lambda \in \Lambda \backslash\{\mu\}}\left(U_{\mu} \cup U_{\lambda}\right)=X .
$$

Since $U_{\mu} \cap\left(\cap_{\lambda \in \Lambda \backslash\{\mu\}} U_{\lambda}\right)=\cap_{\lambda \in \Lambda} U_{\lambda}$ is a closed set by our assumption, $\cap_{\lambda \in \Lambda \backslash\{\mu\}} U_{\lambda}$ is a closed set by Proposition 4.11.

THEOREM 4.13. Let $U_{\lambda}$ be a maximal open set for any element $\lambda$ of $\Lambda$ and $U_{\lambda} \neq U_{\mu}$ for any elements $\lambda$ and $\mu$ of $\Lambda$ with $\lambda \neq \mu$. If $\cap_{\lambda \in \Lambda} U_{\lambda}$ is a closed set, then $\cap_{\lambda \in \Lambda \backslash\{\mu\}} U_{\lambda}$ is a closed set for any element $\mu$ of $\Lambda$.

Proof. By Lemma 2.2(2), we have $U_{\lambda} \cup U_{\mu}=X$ for any elements $\lambda$ and $\mu$ of $\Lambda$ with $\lambda \neq \mu$. By Proposition 4.12, we have that $\cap_{\lambda \in \Lambda \backslash\{\mu\}} U_{\lambda}$ is a closed set.

If the assumption of Proposition 4.12 does not hold, then the condition that $\cap_{\lambda \in \Lambda} U_{\lambda}$ is a closed set does not always imply that $\cap_{\lambda \in \Lambda \backslash\{\mu\}} U_{\lambda}$ is closed. The following is an example.

EXAMPLE 4.14. Let $X=\{a, b, c, d, e\}$ with topology $\theta=\{\varnothing,\{a\},\{d\},\{a, d\}$, $\{b, d\},\{c, d\},\{a, b, d\},\{a, c, d\},\{b, c, d\},\{a, b, c, d\},\{b, c, d, e\}, X\}, U_{1}=\{a\}$, $U_{2}=\{a, b, d\}$, and $U_{3}=\{a, c, d\}$. Then, $U_{1} \cap U_{2} \cap U_{3}=U_{1}$ is a closed set. It follows that $U_{1} \cup U_{2}=U_{2} \neq X, U_{1} \cup U_{3}=U_{3} \neq X, U_{2} \cup U_{3}=\{a, b, c, d\} \neq X$. We see that $U_{2} \cap U_{3}=\{a, d\}$ is not a closed set.

5. More about radicals of maximal open sets. In this section, we study the closure of radicals. We begin with a proposition.

Proposition 5.1. Let $U_{\lambda}$ be a set for any element $\lambda$ of $\Lambda$. If $\mathrm{Cl}\left(\cap_{\lambda \in \Lambda} U_{\lambda}\right)=X$, then $\mathrm{Cl}\left(U_{\lambda}\right)=X$ for any element $\lambda$ of $\Lambda$.

Proof. We see that $X=\mathrm{Cl}\left(\cap_{\lambda \in \Lambda} U_{\lambda}\right) \subset \mathrm{Cl}\left(U_{\lambda}\right)$. It follows that $\mathrm{Cl}\left(U_{\lambda}\right)=X$ for any element $\lambda$ of $\Lambda$.

THEOREM 5.2. Let $U_{\lambda}$ be a maximal open set for any element $\lambda$ of a finite set $\Lambda$. If $\mathrm{Cl}\left(\cap_{\lambda \in \Lambda} U_{\lambda}\right) \neq X$, then there exists an element $\lambda$ of $\Lambda$ such that $\mathrm{Cl}\left(U_{\lambda}\right)=U_{\lambda}$.

Proof. Assume that $\mathrm{Cl}\left(U_{\lambda}\right)=X$ for any element $\lambda$ of $\Lambda$. Let $\mu$ be an element of $\Lambda$. Since $\cap_{\lambda \in \Lambda \backslash\{\mu\}} U_{\lambda}$ is an open set, we have

$$
\begin{aligned}
\mathrm{Cl}\left(\cap_{\lambda \in \Lambda} U_{\lambda}\right) & =\mathrm{Cl}\left(\left(\cap_{\lambda \in \Lambda \backslash\{\mu\}} U_{\lambda}\right) \cap U_{\mu}\right) \supset\left(\cap_{\lambda \in \Lambda \backslash\{\mu\}} U_{\lambda}\right) \cap \mathrm{Cl}\left(U_{\mu}\right) \\
& =\left(\cap_{\lambda \in \Lambda \backslash\{\mu\}} U_{\lambda}\right) \cap X \\
& =\cap_{\lambda \in \Lambda \backslash\{\mu\}} U_{\lambda} .
\end{aligned}
$$


Hence, $\mathrm{Cl}\left(\cap_{\lambda \in \Lambda \backslash\{\mu\}} U_{\lambda}\right) \subset \mathrm{Cl}\left(\cap_{\lambda \in \Lambda} U_{\lambda}\right)$. On the other hand, we see that $\cap_{\lambda \in \Lambda} U_{\lambda} \subset$ $\cap_{\lambda \in \Lambda \backslash\{\mu\}} U_{\lambda}$, and hence $\mathrm{Cl}\left(\cap_{\lambda \in \Lambda} U_{\lambda}\right) \subset \mathrm{Cl}\left(\cap_{\lambda \in \Lambda \backslash\{\mu\}} U_{\lambda}\right)$. It follows that $\mathrm{Cl}\left(\cap_{\lambda \in \Lambda} U_{\lambda}\right)$ $=\operatorname{Cl}\left(\cap_{\lambda \in \Lambda \backslash\{\mu\}} U_{\lambda}\right)$. Then, by induction on the element of $\Lambda$, we see that $\mathrm{Cl}\left(\cap_{\lambda \in \Lambda} U_{\lambda}\right)=\mathrm{Cl}\left(U_{\lambda}\right)=X$ for an element $\lambda$ of $\Lambda$. This contradicts our assumption that $\mathrm{Cl}\left(\cap_{\lambda \in \Lambda} U_{\lambda}\right) \neq X$. Therefore, we see that there exists an element $\lambda$ of $\Lambda$ such that $\mathrm{Cl}\left(U_{\lambda}\right)=U_{\lambda}$.

Theorem 5.2 is not true when $\Lambda$ is not a finite set, as we see by the following example. This example also shows that if $\Lambda$ is not a finite set, then Theorem 4.8 is not always true.

EXAMPLE 5.3. Let $X=\mathbb{R}^{n}$, the $n$-dimensional Euclidean space. Let $U_{x}=$ $X-\{x\}$ for any element $x \in X$. Then, $U_{x}$ is a maximal open set and we have

$$
\mathrm{Cl}\left(\cap_{x \in X} U_{x}\right)=\mathrm{Cl}(\varnothing)=\varnothing \neq X
$$

However, $\mathrm{Cl}\left(U_{x}\right)=X$ for any element $x$ of $X$.

The radicals of maximal open sets have the following outstanding property.

THEOREM 5.4 (the law of radical closure). Let $\Lambda$ be a finite set and $U_{\lambda} a$ maximal open set for each element $\lambda$ of $\Lambda$. Let $\Gamma$ be a subset of $\Lambda$ such that

$$
\begin{array}{ll}
\operatorname{Cl}\left(U_{\lambda}\right)=U_{\lambda} & \text { for any } \lambda \in \Gamma, \\
\operatorname{Cl}\left(U_{\lambda}\right)=X \quad \text { for any } \lambda \in \Lambda \backslash \Gamma .
\end{array}
$$

Then, $\mathrm{Cl}\left(\cap_{\lambda \in \Lambda} U_{\lambda}\right)=\cap_{\lambda \in \Gamma} U_{\lambda}(=X$ if $\Gamma=\varnothing)$.

Proof. If $\Gamma=\varnothing$, then we have the result by Theorem 5.2. Otherwise $\Gamma \neq \varnothing$, and hence we see that

$$
\begin{aligned}
\mathrm{Cl}\left(\cap_{\lambda \in \Lambda} U_{\lambda}\right) & =\operatorname{Cl}\left(\left(\cap_{\lambda \in \Gamma} U_{\lambda}\right) \cap\left(\cap_{\lambda \in \Lambda \backslash \Gamma} U_{\lambda}\right)\right) \supset\left(\cap_{\lambda \in \Gamma} U_{\lambda}\right) \cap \mathrm{Cl}\left(\cap_{\lambda \in \Lambda \backslash \Gamma} U_{\lambda}\right) \\
& =\left(\cap_{\lambda \in \Gamma} U_{\lambda}\right) \cap X=\cap_{\lambda \in \Gamma} U_{\lambda}
\end{aligned}
$$

by Theorem 5.2 and the fact that $\cap_{\lambda \in \Gamma} U_{\lambda}$ is an open set. It follows that $\mathrm{Cl}\left(\cap_{\lambda \in \Lambda} U_{\lambda}\right)=\mathrm{Cl}\left(\mathrm{Cl}\left(\cap_{\lambda \in \Lambda} U_{\lambda}\right)\right) \supset \mathrm{Cl}\left(\cap_{\lambda \in \Gamma} U_{\lambda}\right)$. On the other hand, we see that $\cap_{\lambda \in \Lambda} U_{\lambda} \subset \cap_{\lambda \in \Gamma} U_{\lambda}$, and hence $\mathrm{Cl}\left(\cap_{\lambda \in \Lambda} U_{\lambda}\right) \subset \mathrm{Cl}\left(\cap_{\lambda \in \Gamma} U_{\lambda}\right)$. It follows that $\mathrm{Cl}\left(\cap_{\lambda \in \Lambda} U_{\lambda}\right)=\mathrm{Cl}\left(\cap_{\lambda \in \Gamma} U_{\lambda}\right)$. The radical $\cap_{\lambda \in \Gamma} U_{\lambda}$ is a closed set since $U_{\lambda}$ is a closed set for any $\lambda \in \Gamma$ by our assumption. Therefore, we see that $\mathrm{Cl}\left(\cap_{\lambda \in \Lambda} U_{\lambda}\right)=$ $\cap_{\lambda \in \Gamma} U_{\lambda}$.

As an application of Theorem 5.4, we give another proof of Theorem 4.8.

ANOTHER PROOF OF THEOREM 4.8. Let $\Gamma$ be a subset of $\Lambda$ such that

$$
\begin{array}{ll}
\mathrm{Cl}\left(U_{\lambda}\right)=U_{\lambda} & \text { for any } \lambda \in \Gamma, \\
\mathrm{Cl}\left(U_{\lambda}\right)=X & \text { for any } \lambda \in \Lambda \backslash \Gamma .
\end{array}
$$


We suppose that the radical $\cap_{\lambda \in \Lambda} U_{\lambda}$ is a closed set. We see that $\Gamma \neq \varnothing$ by Theorem 5.4. Then, $\cap_{\lambda \in \Lambda} U_{\lambda}=\operatorname{Cl}\left(\cap_{\lambda \in \Lambda} U_{\lambda}\right)=\cap_{\lambda \in \Gamma} U_{\lambda}$ for the subset $\Gamma$ of $\Lambda$ by Theorem 5.4. Then, we see that $\Lambda=\Gamma$ by Theorem 4.6.

\section{REFERENCES}

[1] N. Jacobson, The radical and semi-simplicity for arbitrary rings, Amer. J. Math. 67 (1945), 300-320.

[2] F. Nakaoka and N. Oda, Some applications of minimal open sets, Int. J. Math. Math. Sci. 27 (2001), no. 8, 471-476.

[3] J. J. Rotman, An Introduction to Homological Algebra, Pure and Applied Mathematics, vol. 85, Academic Press, New York, 1979.

Fumie Nakaoka: Department of Applied Mathematics, Faculty of Sciences, Fukuoka University, Nanakuma, Jonan-ku, Fukuoka 814-0180, Japan

E-mail address: fumi e@fukuoka-u.ac.jp

Nobuyuki Oda: Department of Applied Mathematics, Faculty of Sciences, Fukuoka University, Nanakuma, Jonan-ku, Fukuoka 814-0180, Japan

E-mail address: odanobu@cis. fukuoka-u.ac.jp 


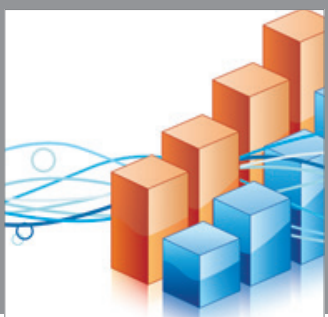

Advances in

Operations Research

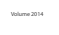

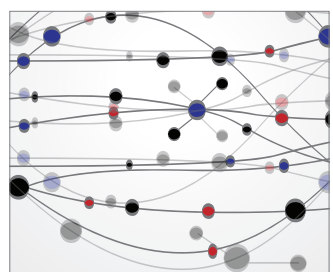

\section{The Scientific} World Journal
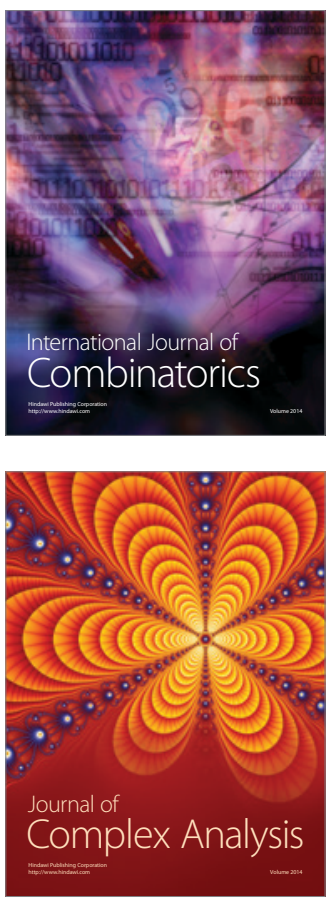

International Journal of

Mathematics and

Mathematical

Sciences
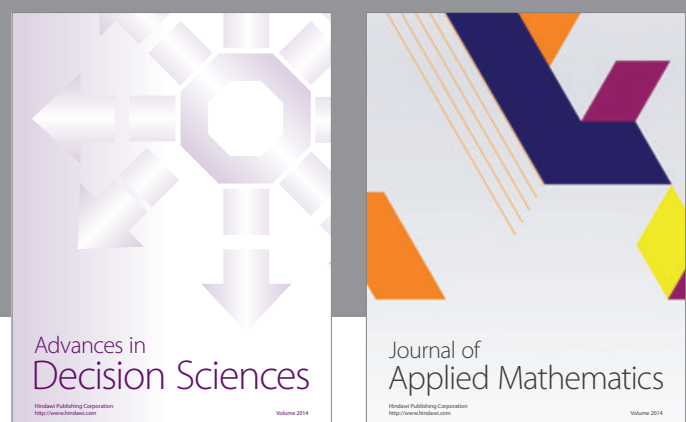

Journal of

Applied Mathematics
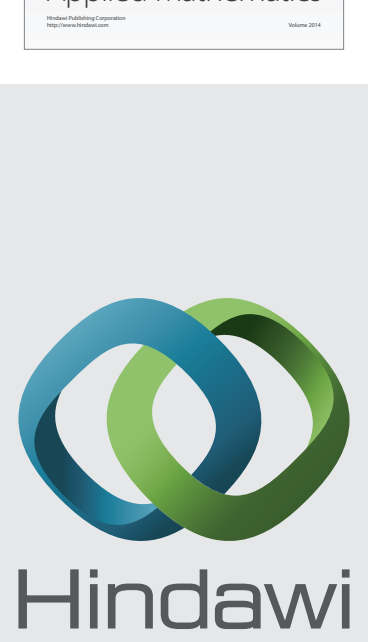

Submit your manuscripts at http://www.hindawi.com
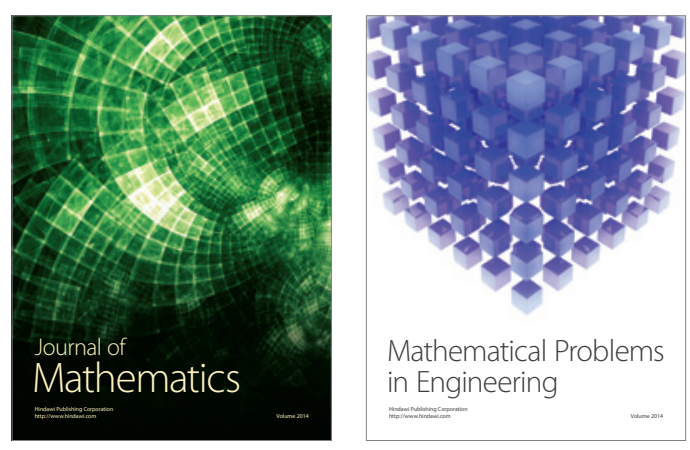

Mathematical Problems in Engineering
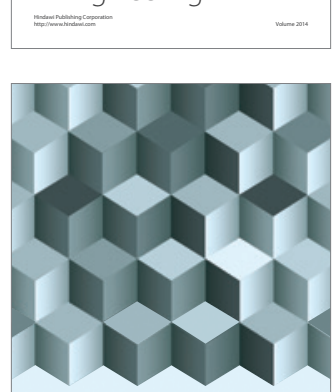

Journal of

Function Spaces
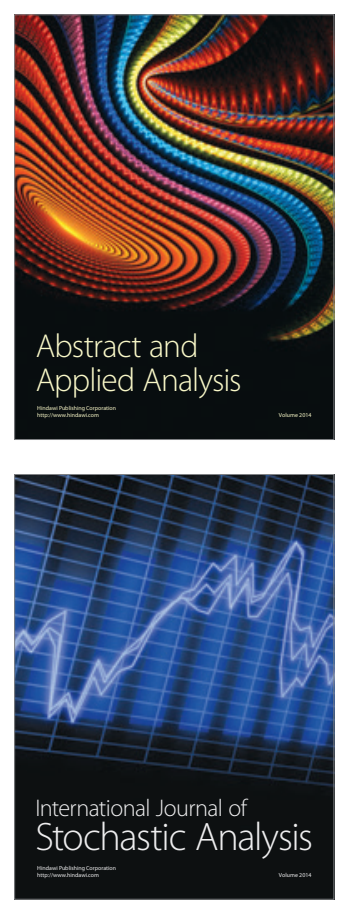

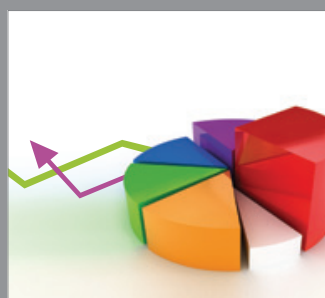

ournal of

Probability and Statistics

Promensencen
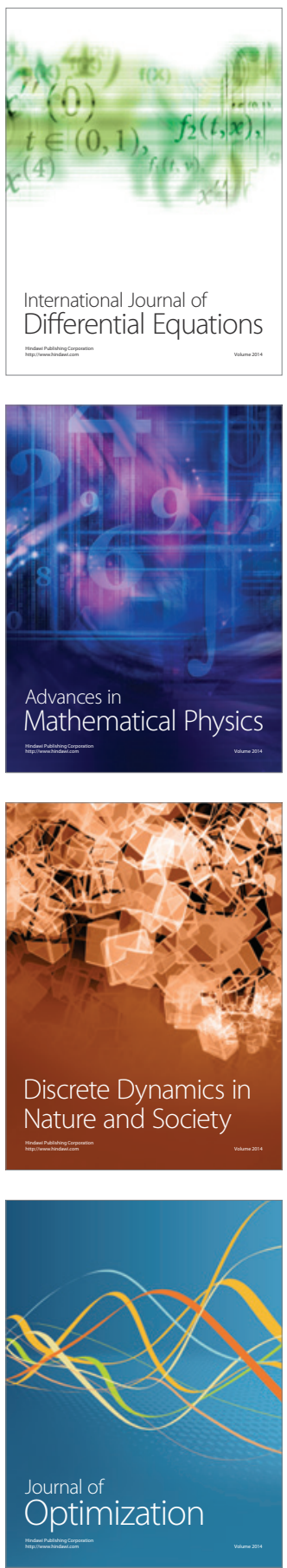